E. Thorup, P. Sindberg Eriksen, K. Rasmussen

Department of Obstetrics and Gynecology

Herlev Hospital

DK-2730 Herlev, Copenhagen, Denmark

\title{
Introduction
}

The blood flow is determined by measuring the diameter of the vessel and the velocity of the blood. The diameter is measured by a linear array scanner and the velocity is measured by a pulsed Doppler.

\section{Measuring set-up}

The measuring set-up includes the following components:

1. Linear array scanner with caliper system, freezing system, and time motion mode.

The pictures are displayed on a TV screen.

2. Hard copy unit for documentation.

3. Bidirectional pulsed Doppler for determination of velocity curves. The Doppler transducer is mounted on the linear array transducer in an angle of $45^{\circ}$.

Because of interference between the ultrasound systems the transducers cannot be used simultaneously. As soon as the vessel is localized and positioned by the linear scanner the pulsed Doppler is switched on and the scanner is switched off.

4. Multichannel paper recorder for velocity curves from the pulsed Doppler.

5. Oscilloscope for display of the maximum velocity curve in order to obtain correct measuring depth.

6. Headset for listening to the Doppler signal.

Determination of the vessel diameter

By the linear scanner a longitudinal section of the vessel is localized. The time motion system is selected and the gain is reduced until the movements of the vessel wall are displayed as two distinct lines on the screen. The picture is frozen and copied by the hard copy unit. Later the copy is enlarged and by using the built-in distance dots the average distance between the lines representing the vessel is determined. on the assumption that the vessel is circular the distance is equal to the average diameter. The distance is determined by planimetry through approximately 4 seconds.

\section{Determination of blood velocity}

The distance from the Doppler transducer and the vessel is measured by the linear scanner. The pulsed Doppler is set to this distance and switched on. By listening to the sound and looking at the velocity curve on the oscilloscope the measuring depth for the pulsed Doppler is adjusted to correct position. Correct position means that the velocity curve is above zero in diastole.

Velocity curves are recorded on the paper recorder and the curves represents maximum-, mean-, and temporal mean velocity of the blood.

Correct angle between the vessel and the Doppler transducer is measured on the hard copy and the velocities are divided by cosine to this angle. The J.Perinat.Med. 12(1984)

Copyright by Walter de Gruyter \& Co., Berlin-New York 
average velocity of the blood is now stated and the average flow in $\mathrm{ml} / \mathrm{min}$. is calculated according:

$$
\text { II/4x (average diameter) }{ }^{2} \mathrm{x} \text { (average velocity) } \mathrm{x} 60 .
$$

\section{Doppler spectrum}

The Doppler effect excists for ultrasonic waves. This effect is a change in the frequency of an ultrasonic wave when the transmitter, the receiver, or the scatterer are moving proportional to each other. Therefore reflections from moving red cells have a different ultrasonic frequency from the transmitted frequency. Ultrasonic Doppler flowmeters responds only to reflections which have experienced a Doppler shift and not at all to the reflections from stationary structures.

When the scatterer is moving with a particular velocity the amount of Doppler shift $\Delta$ f is:

$$
\Delta \mathrm{f}=\frac{2 \mathrm{x} \times \mathrm{v}}{\mathrm{C}} \times \cos \varphi
$$

$f=$ frequency of transmitted ultrasound

$\mathrm{v}=$ velocity of scatter

$c=$ velocity of sound in the tissue

$\Phi=$ angle between sound beam and direction of scatter.

As the red cells are moving with different velocities the Doppler shift will include many different frequencies, the so called Doppler spectrum. By simply listening to the Doppler frequencies it is possible to interpret many points in the dynamics of flow. Determination of the mean frequency of the Doppler spectrum and conversion of the signal to an analog signal is usually performed in a zero-crossing detector. The number of zero-crossings per unit time is proportional to an analog voltage which can be recorded.

A disadvantage of zero-crossing detection is that the output is independent of the amplitude of the audio-signal only at higher input levels. The pulsed Doppler in the measuring set-up used here is based on the relationship between power spectrum for the Doppler spectrum and the velocity distribution for the red cells.

An estimate for the power spectrum is generated and following velocity curves are recorded

- maximum velocity

- mean velocity

- temporal mean velocity.

If the flow profile is like a parabola the flow is laminary and the velocity distribution of the red cells is well defined and rectangulary. This means that the maximum velocity is twice the mean velocity.

By using a frequency analyser Fast Fourier Transform Analyser, FFT, the determination of blood flow is not expected to be better as the troublesome parameter is the diameter. As regards diagnosing and grading of stenoses and other changes which gives rise to changes in the vessel flow a FFT is found to be of the greatest importance.

Use of measuring set-up

By the measuring set-up

- blood flow in $\mathrm{ml} / \mathrm{min}$. can be calculated

- pulsatility index for estimation of the resistance in the circu- 
lation and the elasticity of the vessel can be specified

- estimation of flow profiles during systole and diastole as severe changes from the laminary flow can be stated.

\section{Discussion of results}

The penetration of the real time scanner is $180 \mathrm{~mm}$ and the diameter to be measured is approximately $6 \mathrm{~mm}$. Further the aorta is pulsatile. A suitable average value has to be made and in the formula for calculating blood flow the diameter is squared. Measuring an average diameter is difficult and it has to be done with the greatest patience and care in order to reduce the error in the final result.

The angle between sound beam and vessel has to be measured within $1^{\circ}$. During determination of the velocity curves a filter is used to cut away all information below $100 \mathrm{cps}$. The purpose is to eliminate the movement of the vessel wall as this wall reflects a lot of the ultrasound energy. This reflections again results in a artifact in the Doppler spectrum as regards the red cells.

Estimation of the mean velocity will therefore be too high. It is difficult to state how much, but according to the manufacturer the temporal mean has to be reduced by $2.71 \mathrm{~cm} / \mathrm{sec}$. When a filter of $100 \mathrm{cps}$. is used and the angle is 45 degrees. This assumes a parabolic flow profile. In order to compare results obtained by this estimation system it is of the greatest importance that the influence of the filter, wall movement canceller, is specified in details.

\section{Conclusion}

The measuring set-up has proved to give reliable and reproducible flow in $\mathrm{ml} / \mathrm{min}$. A drawback for the method is that it is too complicated to use from a clinical point of view, especially determination of an average diameter. At the moment the method is not ready for introduction in the clinic but the results obtained justify the need for a method in one way or another.

We suggest that a new method will be based on the relationship between velocity distribution and the power spectrum for the Doppler spectrum in combination with a real time scanner. Location of the vessel is performed by the scanner and the velocity distribution is performed by the pulsed Doppler in combination with a FFT analyser. The results will be displayed as flow profiles and no diameter measuring is necessary. The system can be used in diagnosing abnormal flow conditions, in other words it is a universal system. The physician who have a thorough understanding of the hemodynamics of the circulation will be able to interpret the flow profiles rather fast and without any pain and discomfort for the patient. 